\title{
Pedestrian Road Crossing Behavior Under Mixed Traffic Conditions: A Comparative Study of an Intersection Before and After Implementing Control Measures
}

\author{
Gowri Asaithambi $^{1} \cdot$ Manu O. Kuttan ${ }^{1} \cdot$ Sarath Chandra $^{1}$
}

Received: 20 December 2015/Accepted: 30 May 2016/Published online: 16 June 2016

(C) Springer International Publishing Switzerland 2016

\begin{abstract}
Traffic accidents involving pedestrians have become a major safety problem in most of the developing countries like India due to rapid urbanization, increase in vehicular growth and lack of adherence to traffic regulations by both drivers and pedestrians. The existing roads design does not provide adequate facilities for pedestrians and hence, there exists a constant conflict between the pedestrians and the vehicles in sharing the limited space available on a road. At intersections, pedestrian crossing behavior is even more complex since the vehicles have very little response time to control the vehicles, particularly in urban areas. All these issues are pointing towards the research work required for studying the road crossing behavior of pedestrians at urban intersections. The main motivation of this study is to investigate the changes in pedestrian road crossing behavior of an intersection under mixed traffic conditions before and after signal installation. For this purpose, traffic and pedestrian data were collected from an intersection located in Mangalore city. Different parameters like age, gender, crossing patterns, crossing times, waiting times and crossing speeds of pedestrians, speed and types of vehicles and vehicular flows were extracted from the videos. The study analysis shows that majority of the pedestrians chooses one-step crossing after signal installation since they get sufficient gap to cross the road during the red phase of the signal provided for vehicles.
\end{abstract}

Gowri Asaithambi

gowri_iitm@yahoo.co.in

Manu O. Kuttan

manuokuttan1989@gmail.com

Sarath Chandra

sarathchandra120@gmail.com

1 Department of Civil Engineering, National Institute of Technology, Surathkal, Mangalore, Karnataka, India
The waiting times of the pedestrians were increased after installation of the signal since pedestrians have to wait for gaps until the vehicles get dissipated during the green phase. The 15th percentile crossing speed of pedestrians for both the scenarios were lesser than the crossing speed $(1.2 \mathrm{~m} / \mathrm{s})$ used in pedestrian facility design as per Indian Road Congress (IRC 103). Critical gap of pedestrians has reduced marginally due to the decrease in crossing speeds of pedestrians after installation of the signal. A model was developed considering pedestrian gap size as the dependent variable and pedestrian and traffic characteristics as the independent variables using multiple linear regression (MLR) technique. A total of 468 and 333 accepted gaps of pedestrians were used for modeling the before and after scenarios, respectively. It was found that pedestrian jaywalking behavior is reduced after installation of the signal. The study concludes that after signal installation the factors such as traffic volume, average speed of vehicles and age-group play a predominant role in estimating the pedestrian gap acceptance. This study will be useful for understanding the complex pedestrian road crossing behavior at intersections under mixed traffic conditions. The findings of this paper have great implications for designing pedestrian facilities and also, for devising traffic management measures for pedestrian safety.

Keywords Pedestrian road crossing - Pedestrian gap acceptance $\cdot$ Uncontrolled intersection $\cdot$ Signalized intersection $\cdot$ Control measures $\cdot$ Mixed traffic

\section{Introduction}

Walking is a key non-motorized mode of transport used by pedestrians that connects different components of a multimodal transport network and interfaces with external 
activity areas. The pedestrian is often the most vulnerable road user of all transportation networks users, and frequently, the most overlooked. Traffic accidents involving pedestrians have become a major safety problem in most of the developing countries like India due to rapid urbanization, increase in vehicular growth and lack of adherence to traffic regulations by both drivers and pedestrians. Moreover, traffic is also mixed in nature encompassing vehicles with wide ranging static and dynamic characteristics. All these vehicles move on the same road space occupying any position on the road space based on the availability of free space without complying with any lane discipline. Pedestrians also sharing the same road space which results in severe conflicts with vehicles. In addition to that, in the existing road network designs, footpaths, approaches to bus stops, bus priority lanes, continuous pedestrian paths, lane for slow vehicles like bicycles and rickshaws, etc., have not been included. This often leads to unsafe conditions for pedestrians and slow moving vehicles, and congested conditions for motorized vehicles. As per National crime records bureau, 12,385 pedestrian deaths were reported for the year 2013 in India [1]. More than half of injured and killed pedestrians were young men in the age group of 16-45. At intersections, the complexity of interactions between pedestrian and vehicular traffic is severe. Such conditions are very common on urban roads of medium sized cities in developing countries like India.

The behavior of pedestrians under mixed traffic conditions especially in India is comparatively different from that of other countries. In developed countries, traffic and pedestrian movements are controlled based on certain priorities at uncontrolled intersections whereas in India rules of priority are not fully respected. Even though proper control measures like signals are provided at uncontrolled intersection, pedestrians tentatively decline to use the signals and avoid using pedestrian crossing locations which affect the pedestrian as well as the traffic stream characteristics adversely. In most of the signalized intersections in India, no exclusive phase is provided for pedestrian movement and they generally cross the intersection during the red phase provided for vehicles. Hence, it is clear that pedestrian safety is the major concern of transport planners, traffic engineers and policy makers in India. In India, little attention has been devoted to study pedestrian road crossing behavior and pedestrian flow characteristics, and model them. Moreover, there are only limited studies which focus on the pedestrian road crossing behavior at un-controlled intersection under mixed traffic conditions. Hence, the present study focuses on the road crossing behavior of pedestrians at un-controlled intersection in mixed traffic conditions with the following specific objectives:
1. To identify the different types of crossing patterns of pedestrians at the intersection before and after installation of traffic signal.

2. To analyze and compare the waiting times and crossing speeds of pedestrians with respect to age, gender and crossing patterns before and after signal installation.

3. To develop a multiple linear regression model for the pedestrian gap acceptance by considering pedestrian and traffic characteristics.

To investigate these objectives, traffic and pedestrian data including the pedestrian demographic characteristics were collected from an intersection before and after installation of signal in Mangalore city, India. To achieve the first objective, video data was processed in an image processing software and type of pedestrian movements were noted by visual observation. For the second objective, waiting times, crossing speeds, age, gender and crossing pattern of pedestrians were extracted from the videos before and after installation of signal. Crossing speeds of different pedestrians were compared based on the age, gender and crossing pattern of the pedestrians. For the last objective, a model for the pedestrian gap acceptance was developed by incorporating pedestrian accepted gap size as the dependent variable and age, gender and crossing pattern of pedestrians, traffic volume, types and speeds of vehicles as the independent variables.

The rest of this paper is structured as follows. In the next section, relevant literature on pedestrian crossing behavior under homogenous and mixed traffic conditions is reviewed. Section "Data Collection" discusses about the data collection process and extraction. Detailed discussions on road crossing behavior of pedestrians before and after installation of traffic signal were presented in section "Data Extraction and Analysis". The development of Multiple Linear Regression (MLR) model for modeling pedestrian gap acceptance behavior is discussed in section "Multiple Linear Regression Model" followed by conclusions.

\section{Review of Literature}

This section discusses about the studies carried out to understand the pedestrian road crossing behavior at midblock locations and intersections under homogeneous and mixed traffic conditions. Many studies revealed that age and gender significantly affect the pedestrian behavior and found that males have a tendency to show more hazardous road crossing behavior than females due to less waiting time [2-10]. Models were also developed for pedestrian crossing speeds and crossing times which were helpful for 
designing crosswalk width [11, 12]. Zhang et al. [13] investigated the effects of pedestrian green time, crosswalk length and pedestrian crossing direction on pedestrian walking speed at signalized crosswalk. Studies also found that crossing behavior of the pedestrians were affected by traffic volume and conditions also, due to darkness and weather conditions [14]. The pedestrian crossing behavior was classified into different categories namely, two-gap, risk-taking, two-stage, and walk and look; single stage, and rolling at different facilities $[15,16]$. Studies on pedestrian gap acceptance and critical gap revealed that walking speed and width of the road will govern a pedestrian to accept or reject gaps and there is a margin of $2 \mathrm{~s}$ between lag and gap $[9,17-19]$. Models were also developed to study the size of the traffic gaps accepted by the pedestrians [20].

Few attempts have been made to study the road crossing behavior of pedestrians under mixed traffic conditions. Road crossing behavior of pedestrians with respect to the demographic characteristics has been observed in various studies. Numerous studies have also explored the importance of pedestrian speed at different locations and they outlined that males walk significantly faster than females while crossing the roads [21-25]. Studies on crossing pattern of pedestrians were also carried out by various researchers and they outlined that the pedestrian crossing maneuvers were divided into three different stages namely, single-stage, two-stage and rolling. It was observed that with one-way movement, more number of pedestrians looked for two-stage gaps, whereas single-stage crossing was prevalent on two-way roads [22, 23]. Paul and Rajbonshi [26] suggested three types of gaps for pedestrians namely single-stage, two-stage and rolling for an unsignalized road. Rastogi and Chandra [27] simplified the pedestrian crossing maneuvers into three different stages namely single-stage, two-stage and rolling. It was observed that with one-way movement, more number of pedestrians looked for two-stage gaps whereas single-stage crossing was prevalent on two-way roads.

Rastogi et al. [21] analyzed the variations in the gap acceptance behavior through descriptive and parametric analysis on various types of facilities like two-lane oneway roads, two-lane two-way roads and three lane two-way roads. The effect of land use was also analyzed and the accepted gap was high in commercial land use and low (less than $8 \mathrm{~s}$ ) in educational and recreational land uses. Pawar and Patil [28] carried out a study at four-lane uncontrolled mid-block roads on critical gap and gap acceptance of pedestrians and the authors outlined that temporal as well as spatial gap acceptance was found to be affected by the conflicting vehicle types and the accepted gap size increases with the increase in the size of conflicting vehicle. In order to find the factors influencing the pedestrian road crossing behavior, researchers had developed several models. Kadali and Vedagiri [29] modeled the behavior of pedestrian road crossing at the uncontrolled midblock location in India under mixed traffic condition using multiple linear regression (MLR) technique. They concluded that increase in the pedestrian waiting time at a curb or a median may reduce their patience and lead to increase in rolling gap behavior and accepted gap size will increase when they accept the lag. Kadali et al. [30] examined the pedestrian gap acceptance behavior in a divided mid-block crossing by employing an artificial neural network model for understanding the decision making process. It was found that pedestrian rolling gap, frequency of attempt, vehicular gap size, pedestrian speed condition and vehicle speed had major role in pedestrian gap acceptance. Models for pedestrian crossing behavior for various facilities under homogeneous conditions were also developed by various researchers. Yannis et al. [31] developed a log normal regression and binary logit model for mid-block street crossings in urban areas to examine the pedestrian gap acceptance and decision making to cross the street. Sun et al. [32] developed realistic models for pedestrian motorist interaction at an uncontrolled two lane mid-block crosswalk. Models were separately developed for pedestrian gap acceptance and motorist yielding behavior using binary logit model, critical gap model, and probability based model and discrete probability models. Zhou et al. [33] studied the different pedestrian behavior using multinomial logit model for modeling regular users, late starters, sneakers and partial sneakers at different signalized intersections in China. Hamed [34] developed models for waiting time at the curb side and number of crossing attempts using proportional hazard and Poisson regression models for both divided and undivided mid-block roads. It was found that gender, age, number of children in house hold, crossing frequency, number of people in the group attempting to cross were some of the most significant predictors from the model.

Most of the literature was concentrating on the study of pedestrian's walking and crossing speed for effective design of new pedestrian facilities or to redesign the existing facilities. Few studies revealed that gap acceptance of pedestrians is a function of waiting and crossing time, crossing speed, age, gender, crossing pattern of pedestrians, speeds and types of vehicles. There were only few attempts made to study the crossing behavior of pedestrians at urban uncontrolled intersections. Moreover, comparisons of pedestrian crossing behavior before and after installation of a signal at an uncontrolled intersection were addressed by limited researchers. Hence, the motivation behind this research work is to study and model the pedestrian gap acceptance behavior at an uncontrolled intersection before and after installation of a traffic signal. The inferences from this study will be helpful for controlling the jaywalking 
behavior of pedestrians and also, for designing the new facility/redesigning the existing facilities in order to improve the pedestrian safety.

\section{Data Collection}

Initially, an attempt was made to study the pedestrian road crossing behavior at an uncontrolled intersection in Kankanady, Mangalore city, India. This intersection is a three legged T-intersection having a major approach of $17 \mathrm{~m}$ width and a minor approach of $15 \mathrm{~m}$ width. Pedestrian activities were higher at the minor approach and hence, it was selected for the study. There was no control measures provided either by means of traffic police or by channelization and also, crosswalk was not marked properly before installation of the signal. The traffic and pedestrian data were collected in the month of December 2014 at this intersection using video-graphic method during peak periods of traffic (5.00-6.00 pm) before installation of signals. In the month of February 2015, a fixed time traffic signal has been installed to control the vehicular movements at this intersection with a clearly marked crosswalk. Then, it was decided to study and compare the pedestrian road crossing behavior before and after the signal was installed. Traffic data was collected after installation of signal during peak periods of traffic (5.00-6.00 pm). The study also aims to check whether the control measures adopted for the safety of pedestrians was adequate or not. The photographs of the study section before and after signalization are shown in Fig. 1.

In the traffic signal, total cycle time is $136 \mathrm{~s}$ with $103 \mathrm{~s}$ for red phase and $33 \mathrm{~s}$ for green phase. These phases are exclusively given for vehicles and it is to be noted that there is no separate time allotted for pedestrians in the signal phase and also, there is no exclusive phase for pedestrians. Pedestrians cross the intersection during the red phase (vehicle stop during this time and hence, pedestrians cross the road without any conflict with vehicles). Some of the pedestrians are violating the signals (i.e., crossing the road during green phase given for vehicles) and these violations are not considered in the present study. The major reasons for pedestrian non-compliance to rules are mixed traffic conditions, non-lane discipline, low quality traffic management, no strict enforcement, pedestrian jay walking behavior, etc. The traffic video data covers a total length of $30 \mathrm{~m}$ along longitudinal direction and $15 \mathrm{~m}$ along lateral direction in the study section.

Extraction of necessary data for the study was carried out using Irfanview 4.38 software. From the captured video, JPEG files were obtained for each second of video recording with the help of Snapshot Wizard software. To reduce errors due to parallax effect of cameras, virtual gridlines overlaid on the video images were used. Here, the length of the road was divided into fifteen grids of each $2 \mathrm{~m}$ and width was divided into fifteen blocks of each $1 \mathrm{~m}$. Knowing distances on the ground and corresponding coordinates on the screen, conversion factors (screen to ground) were worked out. These gridlines were used to get the accurate crossing distance of pedestrians following oblique crossing pattern.

Data on pedestrian and traffic characteristics were extracted for every $1 \mathrm{~min}$. The vehicular gap, vehicular characteristics (types and speeds of vehicles) and pedestrian behavioral characteristics were collected at an accuracy of 1 in $25 \mathrm{~s}$. Crossing speeds of different pedestrians were calculated using the known width of the road and extracted crossing time. The accepted traffic gap was calculated as the difference between two time points: at the first point, the pedestrian is just ready to set foot on the

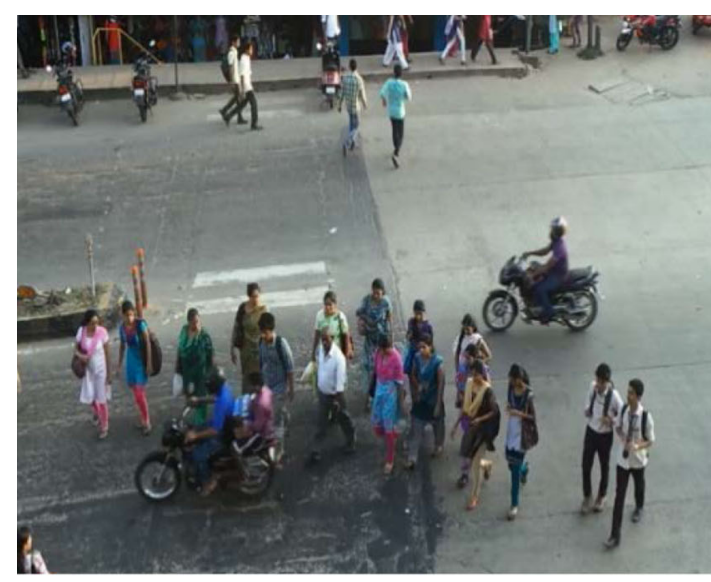

(a)

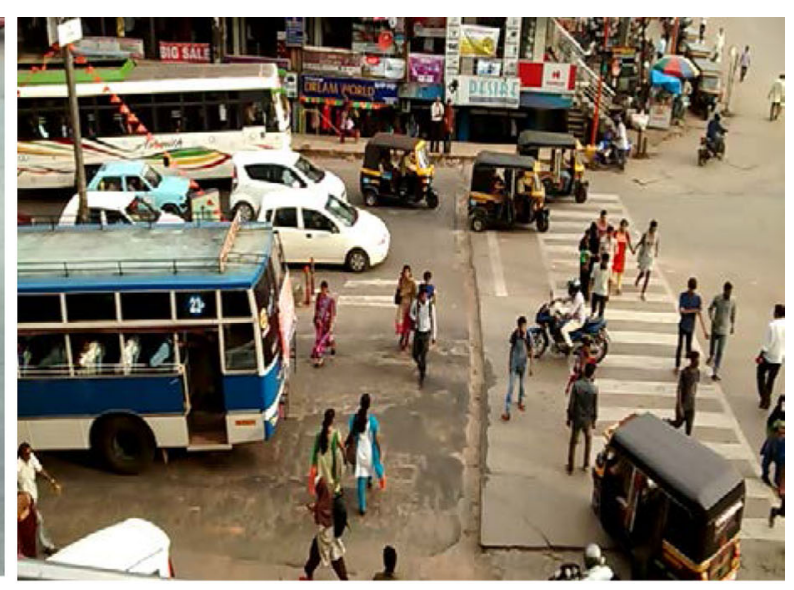

(b)

Fig. 1 Snapshots of the study intersection. a Before installation of signal. b After installation of signal 
street and in the second point, the head of the vehicle has just passed through vertical virtual line indicating the pedestrian's crossing path. Waiting time is the time spent by a pedestrian at the curb or median or both; waiting for suitable gaps to cross the road. Speed of the vehicle is calculated by the known distance of the segment and time taken by the vehicle to traverse the segment when a pedestrian just started to cross the road.

\section{Data Extraction and Analysis}

The data extracted from the videos were analyzed to obtain the individual pedestrian characteristics like gender, age and crossing pattern and traffic stream parameters like composition, speed and vehicular gap accepted by pedestrian. Figure 2 shows the composition of vehicular traffic before and after installation of traffic signal. The pedestrian demographic characteristics such as gender (male and female), age groups (i.e., 10-20, 20-40, 40-50, and above 50 years) and crossing patterns (one-step and two-step) were obtained by visual appearance from the video. The traffic stream parameters were obtained from the videos as explained in section "Data Collection."

\section{Analysis of Crossing Patterns}

Two major crossing patterns of pedestrians were observed in the study intersection:

(a) One-step (pedestrians cross the road without waiting near the median).

(b) Two-step (they cross up to the median in one go and subsequently cross the far side).

One-step and two-step crossings were again subdivided into perpendicular, oblique and mixed crossings based on field observations. When a pedestrian crosses the road in a straight path, it is called perpendicular crossing (PC). Oblique crossings $(\mathrm{OC})$ are those in which pedestrians cross the road in a zig-zag manner. Mixed crossings (MC) are the combination of both perpendicular and oblique type of crossings (pedestrians cross first half of the road in a straight path up to the median and the next half they cross in a zigzag manner and vice versa). The major crossing patterns are explained with the help of a diagram as shown in Fig. 3. The proportion of pedestrians exhibiting different crossing patterns with respect to age group and gender are presented in Table 1. It was found that after improvement, more than $50 \%$ of the pedestrians chooses one-step crossing and hence, the crossing speed was increased by $28 \%$.

\section{Analysis of Waiting Times}

The waiting time of pedestrians at the intersection before and after implementation of a signal was determined. Statistical test $(F$ test) at a significance level of $5 \%$ was performed to check whether the waiting times are different for before and after scenarios and found that they are statistically different. The average waiting times were 4.65 and $7.07 \mathrm{~s}$ for before and after scenarios, respectively.

Pedestrian waiting times were analyzed for different age groups and gender and the variations are shown in Fig. 4. The average waiting time of male pedestrians increased significantly after signals were installed. Male pedestrians are not generally waiting at the curb and median and they have the tendency to roll over the available gaps before installation; but after installation they have to compulsorily wait until the vehicles cross the intersection during the green time. In the case of females, there is no much difference in the waiting times as they always wait for gaps and also more cautious while crossing the road before and after scenarios. From the analysis, it was observed that pedestrians in the age group of 10-20 and above 50 have variations in the waiting times after installation of signal. This is believed to be due to increase in sample size in the age group of 10-20. Hence, when designing the signal, particular attention should be given to the pedestrian waiting times.

\section{Analysis of Crossing Speeds}

Pedestrian crossing speed is the crucial parameter in designing the pedestrian facilities. The average crossing
Fig. 2 Composition of vehicular traffic at the study intersection. a Before installation of signal. b After installation of signal

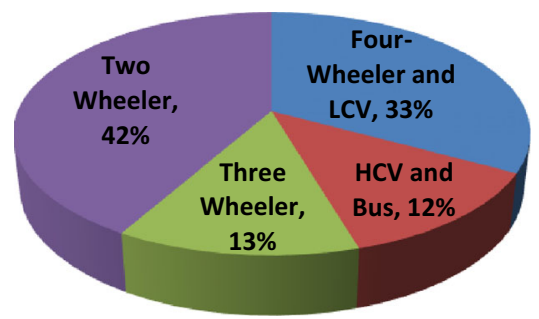

(a)

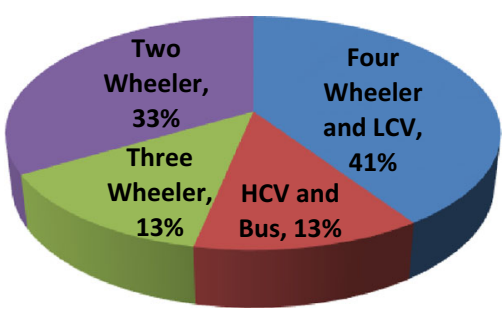

(b) 


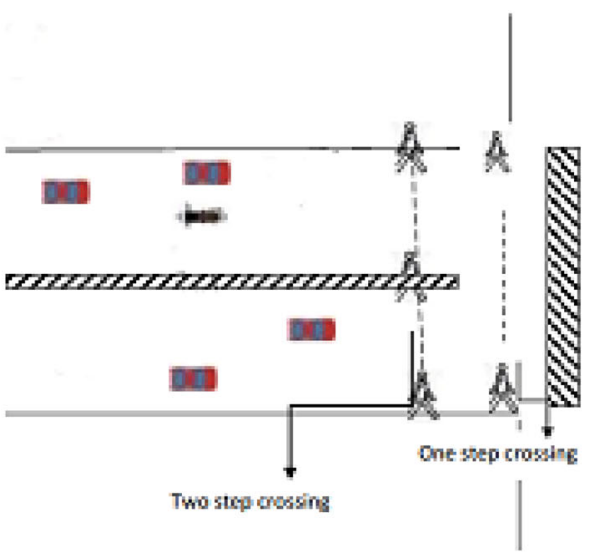

(a)

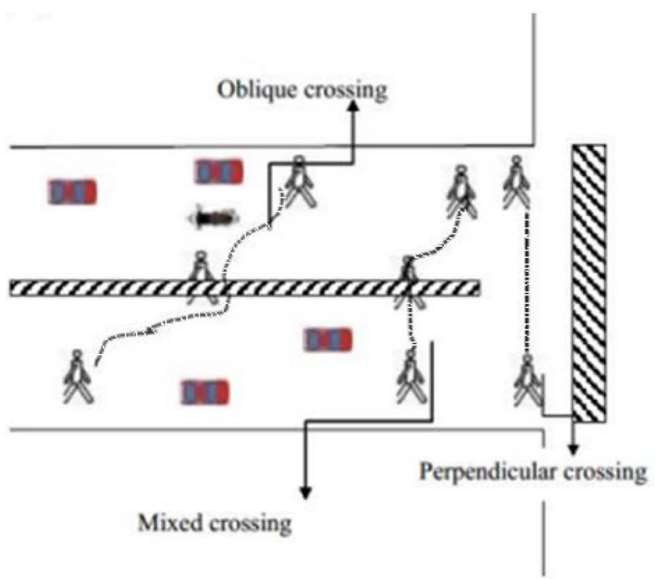

(b)

Fig. 3 Crossing patterns observed in the study location. a Major crossing pattern. b Sub-classification of crossing pattern

Table 1 Details of pedestrians with respect to age group, gender and crossing patterns

Fig. 4 Variation of waiting time based on gender and age group

\begin{tabular}{lll}
\hline & Before signal installation & After signal installation \\
\hline Vehicular flow (veh/h) & 2732 & 2537 \\
Pedestrian flow (ped/h) & 285 & 240 \\
Female & $48 \%$ & $38 \%$ \\
Male & $52 \%$ & $62 \%$ \\
Age group & & \\
$10-20$ & $12 \%$ & $38 \%$ \\
$20-40$ & $59 \%$ & $36 \%$ \\
$40-50$ & $15 \%$ & $18 \%$ \\
$>50$ & $14 \%$ & $8 \%$ \\
Crossing type & & \\
One-step crossing & $15 \%$ & \\
PC & $7 \%$ & $31 \%$ \\
OC & $16 \%$ & $14 \%$ \\
MC & $38 \%$ & $16 \%$ \\
Overall & & $61 \%$ \\
Two-step crossing & $35 \%$ & $17 \%$ \\
PC & $11 \%$ & $6 \%$ \\
OC & $16 \%$ & $16 \%$ \\
MC & $62 \%$ & $39 \%$ \\
Overall & & \\
\hline
\end{tabular}

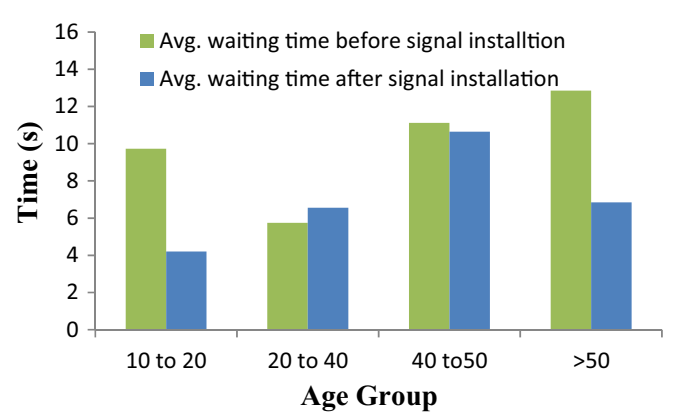


speed of pedestrians was found to decrease by $23 \%$ (from 1.37 to $1.05 \mathrm{~m} / \mathrm{s}$ ) after signal installation. The average crossing speeds were higher before installation since most of the pedestrians are crossing the road in two-step. But, after signal installation, majority of them chose one-stage crossing $(61 \%)$ and hence, the crossing speeds were lesser. The average crossing speeds of the pedestrians with respect to their age group, gender and crossing pattern are presented in Table 2. Statistical test at (5\% level of significance) proves that there is significant difference in the average crossing speeds of pedestrians with respect to age group, gender and crossing pattern before and after installation of the signal. In all the cases, average crossing speeds were decreased considerably after installation of the signal. This decrease in the crossing speeds can be an indication of the safety measures adopted in the study location which helps the pedestrians to cross the intersection more leisurely and in a relaxed manner. The results of average crossing speeds after signal installation were comparable with Jain et al. [23].

Percentile speeds of pedestrians (15th, 50th, and 85th) before and after signal installation were also analyzed using cumulative frequency distribution curves. It was observed that 15 th percentile speeds of pedestrians were approximately 0.7 and $0.5 \mathrm{~m} / \mathrm{s}$ before and after installation of signal, respectively. Generally, 15th percentile speed of the pedestrian crossing speed is taken as the design speed for designing the pedestrian facility. These values are lesser than the design speed $(1.2 \mathrm{~m} / \mathrm{s})$ suggested by IRC 103 [35].

The percentile speeds for all types of crossing patterns decreases after signal installation. This may be due to the increase in the proportion of one-step crossing (without waiting near the median). But in the case of before improvement, pedestrians have to accept even the smaller gaps to avoid conflicts with vehicles and hence, the speeds are higher. These crossing speeds for different crossing patterns will be useful when designing an exclusive pedestrian phase in a traffic signal.

\section{Analysis of Gap Acceptance and Critical Gap}

Gap acceptance by the pedestrian plays a crucial role in determining the safety of pedestrians. Lesser the gap size more will be the accident risk to the pedestrian. The pedestrian gap acceptance can be quantified with respect to the minimum threshold distance in terms of corresponding time based on the speed of the nearest vehicle. Hence, the present study also focuses on estimation of gaps between a pedestrian crossing position at an intersection and the approaching vehicle, which are either accepted or rejected by the pedestrian.

From the analysis, it was found that, the average accepted gap for the pedestrians was 4.21 and $3.50 \mathrm{~s}$ for before and after scenarios, respectively. The decrease in the accepted gap size of the pedestrians also underlines the importance of providing an exclusive pedestrian phase in the signal. This also indicates that pedestrians have become more safety conscious after signals were installed since they have to wait until the vehicles cross the intersection during the green phase.

Pedestrian's time gap data were also classified based on gender. It was found that, the average gap accepted by females and males are 4.6 and $4.13 \mathrm{~s}$, respectively before improvement. These values were 3.75 and $3.49 \mathrm{~s}$, respectively after improvement. The female pedestrians are more safety conscious while crossing the road before and after signal installation.

Pedestrian critical gap is affected by several factors and it also plays a major role from the safety point of view. The minimum average time gap that is accepted by half of all
Table 2 Average crossing speeds based on age group, gender and crossing pattern

\begin{tabular}{lll}
\hline & \multicolumn{2}{l}{ Crossing speed $(\mathrm{m} / \mathrm{s})$} \\
\cline { 2 - 3 } & Before installation of signal & After installation of signal \\
\hline Age group & & \\
$10-20$ & 1.47 & 1.13 \\
$20-40$ & 1.38 & 1.1 \\
$20-40$ & 1.21 & 1.01 \\
$>50$ & 1.07 & 0.98 \\
Gender & & \\
Male & 1.37 & 1.12 \\
Female & 1.1 & 0.97 \\
Crossing pattern & & \\
Perpendicular & 1.43 & 1.06 \\
Oblique & 1.38 & 1.10 \\
Mixed & 1.31 & 1.02 \\
\hline
\end{tabular}


pedestrians to cross the road safely (or) the gap which is just equal to the crossing time of a pedestrian is termed as critical gap. Cumulative distribution curves were plotted for gap acceptance $(\mathrm{Fa})$ and crossing time $(\mathrm{Ft})$ of pedestrians and the intersection of the curves [Fa and (1-Ft)] gives the critical gap (Rafts method) as shown in Fig. 5. The critical gap was found to be 5.8 and $5.3 \mathrm{~s}$ for before and after scenarios, respectively. The decrease in the critical gap was due to the decrease in the crossing speed of the pedestrians as they are not being influenced by the moving vehicles after improvement.

\section{Comparison of Pedestrian Road Crossing Behavior Before and After Installation of Signal}

It was observed that there was a significant difference between the pedestrian characteristics before and after installation of signal. The crossing speeds of the pedestrians were higher because pedestrians were taking risk while crossing the road before improvement. It was also found that $61 \%$ of the pedestrians are following one-stage crossing pattern after installation of the signal. This is because before signal installation, pedestrians were crossing through available gaps, but after installation, they have to compulsorily wait until the vehicles get dissipated during green phase. Also, it was observed that higher proportion of pedestrians utilizes crosswalk for crossing of intersection safely after signal installation.

\section{Multiple Linear Regression Model}

In the present study, two different models were developed to predict the pedestrian gap acceptance behavior using multiple linear regression (MLR) model. In an intersection, whether it is a controlled or an uncontrolled intersection, pedestrian crosses the road based on available gaps. Pedestrian gap acceptance has to be modeled in order to compare the time available for crossing and time taken for crossing and also to determine the possibility of safe crossing. Generally, after arriving at curb or median, pedestrian needs to scan the vehicular gaps for crossing the road. The minimum accepted vehicular time gaps by pedestrian have been modeled by considering pedestrian behavioral characteristics and traffic stream characteristics. The pedestrian age (age), gender (gdr), crossing pattern (csp), traffic volume (tv), vehicle type (vt) and vehicle average speed (vas) were considered as the independent variables and logarithm of the accepted gap size was considered as the dependent variable. Traffic in Indian cities comprises of different types of vehicles and hence, traffic volume is expressed in terms of PCU by considering equivalent PCU values of IRC 106 [36] for each type of vehicle. The PCU values considered for twowheeler, car, auto, light commercial vehicles and buses are $0.75,1.0,2.0,2.0$ and 3.7 , respectively. A partial correlation matrix was done to check whether the dependent and independent variables are correlated with each other. It was observed that the correlation coefficient value between the dependent and independent variables is more than 0.5 and the correlation coefficient values among the independent variables is ranging from -0.5 to 0.5. Also, from significance testing it was found that the $p$ values of these variables are lesser than 0.05 . It was inferred that there is a significant correlation between the dependent variable and all the independent variables and hence, these variables were selected for developing the models.

Table 3 shows the summary of variables and their description used for MLR model, $t$ test was conducted to test whether there is any difference between the samples for pedestrian accepted gap size for before and after installation of the signal $\left(\mathrm{t}_{\text {critical }}=1.96\right)$. It was found that the samples used for the models were statistically significant.
Fig. 5 Critical gap of pedestrians. a Before installation of signal. b After installation of signal

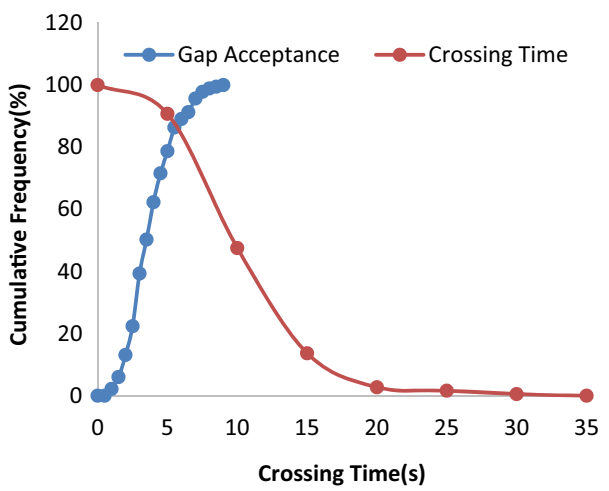

(a)

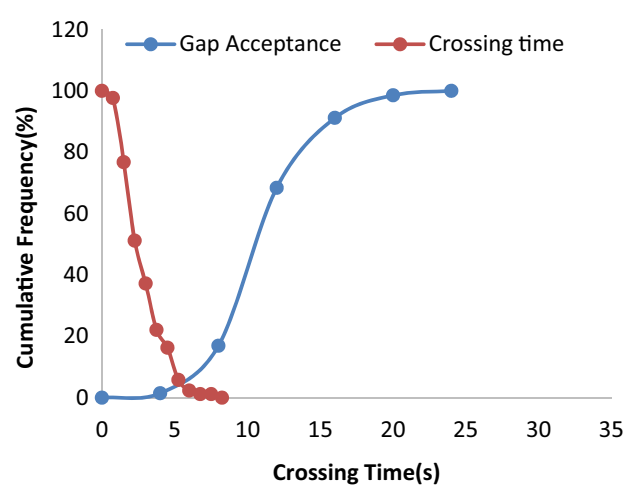

(b) 
Table 3 Types of independent variables and their unit

\begin{tabular}{lll}
\hline Variable & Type of variable & Unit or code \\
\hline Age group & Discrete & $0: 10-20$ \\
& & $1: 20-40$ \\
& & $2: 40-50$ \\
& $3:>50$ \\
Gender & Discrete & $0:$ Male \\
& & 1: Female \\
Crossing pattern & Discrete & $0:$ Perpendicular \\
& & 1: Oblique \\
Traffic volume & 2: Mixed \\
Vehicle type & Continuous & In PCU/hr \\
& Discrete & 0: TW \\
& & 1: Auto-rickshaw \\
Average speed of vehicles & & 2: Car and light commercial vehicle \\
\hline
\end{tabular}

In probability theory, a log-normal (or lognormal) distribution is a continuous probability distribution of a random variable whose logarithm is normally distributed. Thus, if the random variable $\mathrm{X}$ is log-normally distributed, then $\mathrm{Y}=\log \mathrm{X}$ has a normal distribution. The pedestrian gaps follow lognormal distribution more accurately which implies that log gap follows normal distribution [29, 31]. As normal distribution gives best result for random data like gaps, log gap is introduced for modeling. The general model framework is given below:

$\log ($ Gapsize $)=\beta_{0}+\beta_{1} X_{1}+\beta_{2} X_{2}+\beta_{3} X_{3}+\cdots+\beta_{n} X_{n}$

where, Gap size $=$ Accepted gaps; $\beta_{0}=$ constant, $X_{1-n}$ $=$ explanatory variables; $\beta_{1-\mathrm{n}}=$ estimated parameters from the model.

\section{Model Results}

Lognormal regression model was developed using Statistical Package, SPSS to find out the minimum accepted vehicular gap size due to pedestrian road crossing behavior for both scenarios. For developing models, a total of 468 and 333 data points (accepted gaps) were used for before and after scenarios, respectively. For model calibration, $75 \%$ data were considered and remaining $25 \%$ data were used for validation. The descriptive statistics of MLR test for before and after signalization is summarized in Table 4. The calibrated $R^{2}$ value were obtained as 0.749 and 0.839 for before and after scenarios, respectively. The MLR equations for gap size before and after installation of signal can be written as:

$$
\begin{aligned}
\log (\text { Gapsize })_{B I}= & 0.3022+(0.0263) \times \operatorname{age}-(0.0246) \\
& \times \boldsymbol{g d r}+(0.0225) \times \boldsymbol{c s p}+(0.209) \times \boldsymbol{v t} \\
& +(0.0007) \times \boldsymbol{v a s}+(0.0001) \times \boldsymbol{t} \boldsymbol{v}
\end{aligned}
$$

$$
\begin{aligned}
\log (\text { Gapsize })_{A I}= & 0.483-(0.0008) \times \operatorname{age}+(0.0002) \\
& \times \boldsymbol{g d r}+(0.0036) \times \boldsymbol{c s p}+(0.192) \times \boldsymbol{v t} \\
& -(0.0110) \times v a s+(0.0008) \times \boldsymbol{t} \boldsymbol{v}
\end{aligned}
$$

where $\log (\text { Gapsize })_{B I}=$ Accepted gaps for before improvement $\log (\text { Gapsize })_{A I}=$ Accepted gaps for after improvement.

After installation of the signal, gender has positive impact on gap size compared to before improvement. This may be due to the increased proportion of male after improvement $(62 \%)$. After installation, age group has negative impact on speed. This is believed to be due to increase in the proportion of pedestrians in age group of 10-20. This age group generally has higher crossing speeds and hence, the gap size reduces. Average vehicular speed has negative impact on gap size after installation of signal. This shows that pedestrians cross the road during red time provided for vehicles and they are not restricted by vehicular movements and hence, the gap size was reduced. However, in the case of before installation of signal, the vehicles are always in motion and hence, a positive trend was obtained. All the coefficients of the model are logical and reasonable. The graph was plotted between observed and predicted values and a valid $R^{2}$ value has been found (Fig. 6). The results proved that the developed MLR model has better prediction capabilities for estimating the mini- 
Table 4 Gap selection model results

\begin{tabular}{|c|c|c|c|c|c|c|c|c|}
\hline \multirow[t]{2}{*}{ Variable } & \multicolumn{4}{|c|}{ Before signal installation } & \multicolumn{4}{|c|}{ After signal installation } \\
\hline & $\beta$ coefficient & Standard error & $T$ value & $p$ value & $\beta$ coefficient & Standard error & $T$ value & $p$ value \\
\hline Constant & 0.302 & 0.059 & 5.117 & 0.001 & 0.483 & 0.094 & 4.489 & 0.014 \\
\hline Age (age) & 0.026 & 0.010 & 2.403 & 0.001 & -0.0008 & 0.011 & -6.069 & 0.004 \\
\hline Gender (gdr) & -0.024 & 0.016 & -3.48 & 0.001 & 0.0002 & 0.019 & 5.012 & 0.009 \\
\hline Crossing pattern (csp) & 0.022 & 0.011 & 6.942 & 0.005 & 0.003 & 0.013 & 4.278 & 0.007 \\
\hline Vehicle type (vt) & 0.209 & 0.011 & 12.76 & 0.002 & 0.192 & 0.030 & 6.414 & 0.003 \\
\hline Average vehicle speed (vas) & 0.0007 & 0.002 & -3.32 & 0.001 & -0.011 & 0.010 & -4.091 & 0.002 \\
\hline Traffic volume (tv) & 0.0001 & 0.0006 & 4.283 & 0.002 & 0.0008 & 0.0005 & 3.463 & 0.001 \\
\hline
\end{tabular}

Fig. 6 Validation of pedestrian gap acceptance model. a Before installation of signal. b After installation of signal

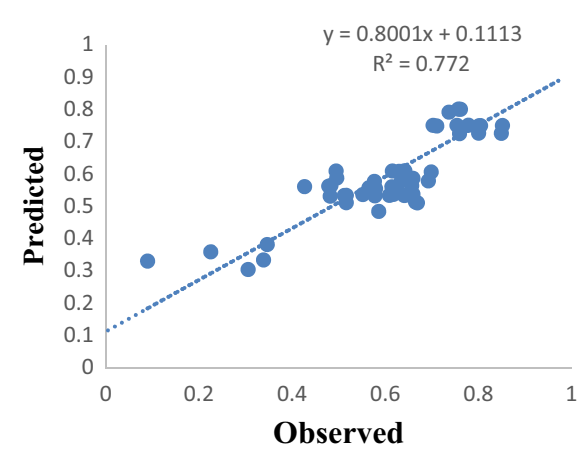

(a)

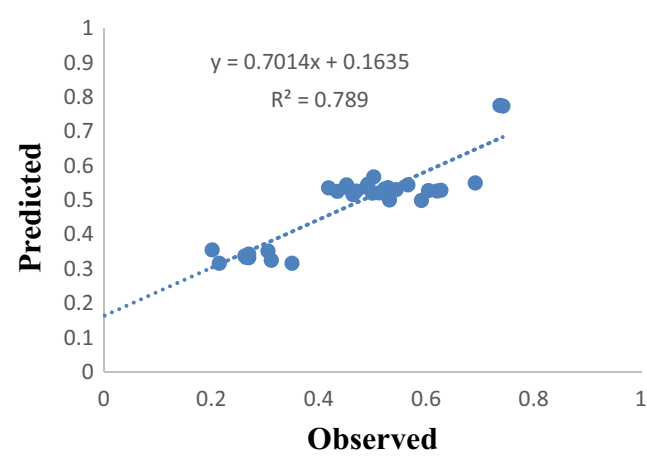

(b) mum vehicular gap size due to pedestrian road crossing behavior.

\section{Conclusions}

The present study made a comparison of pedestrian's road crossing behavior before and after installation of a fixed time signal at an uncontrolled intersection under mixed traffic and non-lane discipline traffic conditions. For this purpose, traffic data were collected at an intersection located in Mangalore city before and after installation of signal. Various parameters like pedestrian age, gender and crossing pattern, waiting times, crossing speeds and gap size were extracted and analyzed. A Multiple Linear Regression (MLR) model was developed in order to examine the effect of various parameters on the size of gaps accepted by pedestrians. The key conclusions arising out of this study are:

- Two types of major crossing patterns were observed in the location such as one-step and two-step crossing. The patterns were sub-classified into three different types such as perpendicular, oblique and mixed movements. After installation of signal, majority of pedestrians chooses one-step crossing (without waiting near the median) since they are able to cross the road without conflicting with vehicles.

- The waiting times of pedestrians were increased by $52 \%$ (from 4.65 to $7.07 \mathrm{~s}$ ) after installation of the signal. This is because pedestrians have to wait until the vehicles get dissipated from the signal during the green phase.

- Female pedestrians were found to cross the road at a slower rate than males in both the scenarios due to their safety concern.

- The average crossing speed after installation of the signal was reduced by $23 \%$ (from 1.37 to $1.05 \mathrm{~m} / \mathrm{s}$ ). This is because majority of the pedestrians chooses one-step crossings after the signals was installed which decreases the speed of pedestrians.

- It was observed that 15 th percentile crossing speed of all pedestrians is higher $(0.73 \mathrm{~m} / \mathrm{s})$ in the case of before improvement compared to after improvement $(0.52 \mathrm{~m} /$ $\mathrm{s})$. Both the values are lesser than the crossing speed $(1.2 \mathrm{~m} / \mathrm{s})$ used in pedestrian facility design as per Indian Road Congress (IRC 103).

- The gaps accepted by males and females decreased after installation of signals. Men appear to take fewer risks than women as they generally accept larger gaps. 
- Due to the decrease in the crossing speed of the pedestrians after installation of the signals, the critical gap was reduced marginally from 5.8 to $5.3 \mathrm{~s}$.

- It was found that the accepted gap size depends on the age, gender and crossing pattern of pedestrian, speed of incoming vehicle, vehicle type and traffic volume.

The study results clearly bring out the variations in crossing behavior of pedestrians after implementing a traffic signal at an intersection. The outcome of the study revealed that traffic signal along with the adequate width of crosswalk has improved the safety of pedestrians crossing the intersection. It is believed that the developed models and study findings may be quite useful to the policy makers to regulate pedestrian jaywalking behavior at urban intersections. The findings will also be helpful to devise traffic management strategies at the intersection to avoid conflicts between pedestrians and traffic. The analysis of crossing pattern will be helpful for planning and designing the crosswalk facilities for improving the safety of pedestrians. The study of crossing speeds and waiting times of pedestrians may help the traffic engineers to design an exclusive pedestrian phase in a signal if it is provided in future. It is therefore important to plan and provide pedestrian facilities in accordance to the observed pedestrian behavior rather than relying on the standard norms or specifications.

In this study, pedestrian's age was considered based on the physical appearance and also, speeds of the vehicles are only considered within the crosswalk area due to limited field of view of camera. The findings of the current study were limited to a single intersection. It is believed that the developed model can be applicable to other study locations which have similar traffic and geometric characteristics. However, further case studies and scenario analysis have to be done to generalize the findings of the present study.

\section{References}

1. Special Report. Pedestrian Safety. NIMHANS BISP Fact Sheet. http://www.nimhans.kar.nic.in/epidemiology/epidem_who2.htm. Accessed 16 Sept 2015

2. Oxley J, Fildes B, Ihsen E, Charlton J, Day R (1997) Differences in traffic judgments between young and old adult pedestrians. Accid Anal Prev 29(6):839-847

3. Khan FM, Jawaid M, Chotani H, Luby S (1999) Pedestrian environment and behaviour in Karachi, Pakistan. Accid Anal Prev 31(4):335-339

4. Fitzpatrick K, Brewer MA, Turner S (2006) Another look at pedestrian walking speed. Transp Res Rec 1982:21-29

5. Shahin MM (2006) Pedestrian behavior with mixed traffic in developing countries. Traffic Eng Control 47(8):303-309

6. Tiwari G, Bangdiwala S, Saraswat A, Gaurav S (2007) Survival analysis: pedestrian risk exposure at signalized intersections. Transp Res Part F 10(2):77-89
7. Oxley J, Fildes B, Ihsen E, Charlton J, Day R (2005) Crossing roads safely: an experimental study of age differences in gap selection bypedestrians. Accid Anal Prev 37(5):962-971

8. Lobjois R, Cavallo V (2006) Age-related differences in streetcrossing decisions: the effects of vehicle speed and time constraints on gap selection in an estimation task. Accid Anal Prev 39(5):934-943

9. Das S, Mansk CF, Manuszak MD (2005) Walk or wait? An empirical analysis of street crossing decisions. J Appl Econom 20(4):529-548

10. Khan FM, Jawaid M, Chotani H, Luby S (1999) Pedestrian environment and behavior in Karachi, Pakistan. Accid Anal Prev 38(4):335-339

11. Jiangang S, Yanyan C, Futian R, Jian R (2007) Research on pedestrian behaviors and traffic characteristics at unsignalized midblock crosswalk: a case study in Beijing. Transp Res Rec 2038:23-33

12. Alhajyaseen W, Nakamura H (2009) A methodology for modeling pedestrian platoon discharge and crossing times at signalized crosswalks. In Proceedings of the 88th transportation research board annual meeting, Washington, DC USA

13. Zhang X, Chen P, Nakamura H, Asano M (2013) Modelling pedestrian walking speed at signalized crosswalk considering crosswalk length and signal timing. In: Proceedings of the 10th International Conference of the Eastern Asia Society for Transportation Studies, Taipei, Taiwan

14. Andrew HW (1991) Factor's influencing pedestrian cautiousness in crossing streets. J Soc Psychol 131(3):367-372

15. Song L, Dunne MC, Black JA (1993) Models of delay and accident risk to pedestrians. In: Proceedings of the 12th international symposium on transportation and traffic flow theory, Berkeley, California

16. Brewer AM, Fitzpatrick K, Whitacre AJ, Lord D (2006) Exploration of pedestrian gap-acceptance behavior at selected locations. J Transp Res Rec 1982:132-140

17. Moore RL (1953) Pedestrian choice and judgment. J Oper Res Soc 4(1):3-10

18. Cohen J, Dearnaley EJ, Hansel CEM (1995) The risk taken in crossing a road. J Oper Res Soc 6(2):120-128

19. Zhao J, Wu J (2003) Analysis of pedestrian behavior with mixed traffic flow at intersection. Intell Transp Sys J IEEE 4(1):323-327

20. Serag MS (2014) Modeling pedestrian road crossing at uncontrolled mid-block locations in developing countries. Int J Civ Struct Eng 4(3):274-286

21. Rastogi R, Chandra S, Vamsheedhar J, Das VR (2011) Parametric study of pedestrian speeds at mid-block crossings. J Urban Plan Dev ASCE 137(4):381-389

22. Chandra S, Rajat R, Das VR (2013) Descriptive and parametric analysis of pedestrian gap acceptance in mixed traffic conditions. J Civ Eng 18(1):284-293

23. Jain A, Gupta A, Rastogi R (2014) Pedestrian crossing behaviour analysis at intersection. Int J Traffic Trans Eng 4(1):103-116

24. Raj kumar T, Ravi Shankar KVR (2014) Pedestrian crossing behavior analysis over space and time. In: Proceedings of the 11th transportation planning and implementation methodologies for developing countries, IIT Bombay, India

25. Marisamynathan S, Vedagiri P (2013) Modeling pedestrian delay at signalized intersection crosswalks under mixed traffic condition. Proc-Soc Behav Sci 104:708-717

26. Paul M, Rajbonshi P (2014) A comprehensive review on pedestrian gap acceptance at unsignalized road. Int J Eng Res Technol 11(3):325-328

27. Rastogi R, Chandra S (2013) Descriptive and parametric analysis of pedestrian gap acceptance in mixed traffic conditions. KSCE J Civ Eng 18(1):284-293 
28. Pawar DS, Patil GR (2015) Pedestrian temporal and spatial gap acceptance at mid-block Street crossing in developing world. J Saf Res 52(1):39-46

29. Kadali BR, Vedagiri P (2013) Modeling pedestrian road crossing behavior under mixed traffic condition. Eur Trans 55(3):1-7

30. Kadali BR, Rathi N, Perumal V (2014) Evaluation of pedestrian mid-block crossing behavior using artificial neural network. J Traffic Transp Eng 1(2):111-119

31. Yannis G, Papadimitriou E, Theofilatos A (2010) Pedestrian gap acceptance for mid-block street crossing. World transport research-proceedings from the 12th world conference on transport research, 1-11

32. Sun D, Ukkursuri SK, Benekohal RF, Waller ST (2003) Modeling of motorist pedestrian interaction at uncontrolled mid block crosswalk. In: 82ndTRB Annual Meeting, Transportation Research Board, National Research Council, Washington, DC

33. Zhou ZP, Liu YS, Wang W, Zhang Y (2013) Multinomial logit model of pedestrian crossing behaviours at signalized intersections. Discret Dyn Nat Soc 2013:172726. doi:10.1155/2013/ 172726

34. Hamed MM (2000) Analysis of pedestrian behavior at pedestrian crossings. Saf Sci 38:63-82

35. Guidelines for pedestrian facilities (2012) IRC 103. Indian Road Congress, India

36. Guidelines for Capacity of Urban Roads in Plain Areas (1990) IRC 106. Indian Road Congress, India 